\title{
Formigas como bioindicadores da qualidade do solo em sistema integrado lavoura- pecuária
}

\author{
Ants as bioindicators of soil quality in integrated crop-livestock system

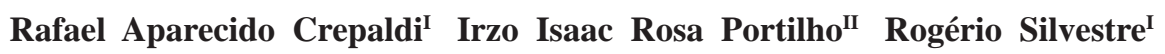 \\ Fábio Martins Mercante ${ }^{\mathrm{III}}$
}

\section{RESUMO}

\begin{abstract}
O objetivo deste trabalho foi avaliar a comunidade de formigas em sistemas envolvendo a rotação entre lavoura e pecuária, sob plantio direto, comparado a outros sistemas de manejo e com sistemas naturais da região. O estudo foi conduzido no Município de Dourados, MS, sob Argissolo Vermelho distroférrico típico, num sistema integrado lavoura-pecuária, em plantio direto, rotacionado a cada dois anos. Outros sistemas de manejo avaliados, para comparação, incluíram o sistema convencional (aração e gradagem), sistema plantio direto $e$ pastagem cultivada continuamente com Brachiaria decumbens, além de dois fragmentos de vegetação nativa, nos biomas Cerrado e Floresta Semidecídua. A coleta de solo foi realizada no período chuvoso (safra 2009/2010), sendo as amostragens de serapilheira realizadas com a utilização de extratores do tipo mini-Winkler, seguindo o protocolo ALL (Ants of the Leaf Litter). Os resultados indicaram morfoespécies de Strumygenys sp. e Hypoponera $s p$. como potencial bioindicadores de qualidade do solo. O estudo demonstrou que a importância da manutenção de fragmentos florestais (Cerrado e Floresta Semidecídua) e implementação de sistemas integrados, tais como lavoura-pecuária, são menos impactantes para a mesofauna de solo e contribuem para a conservação da diversidade de formigas e manutenção da qualidade do solo.
\end{abstract}

Palavras-chave: biodiversidade, agroecossistemas, manejo do solo.

\section{ABSTRACT}

The aim of this study was to evaluate the ant community in different systems involving the crop rotations between livestock, under no-tillage, compared to other management systems and natural systems of the Mid-Western region. The study was carried out in the Dourados, Mato Grosso do Sul State, Brazil, on a Typic Hapludox soil, in parcels including crop-livestock rotation production system under no-tillage, rotated every two years, no-tillage crop system, succession of crops under conventional tillage, continuous pasture and two fragments of native vegetation, Cerrado and Semideciduous Forest. The samples of soil were conducted during the rainy season (2009/2010), and litter sampling performed with the use of mini-Winkler extractors, following the protocol ALL (Ants of the Leaf Litter). The results indicated that the morphospecies of Strumygenys sp. and Hypoponera $s p$. as potential bioindicators of soil quality, depending on their frequency changes in response to different systems observed. The study have demonstrated the importance of maintaining forest fragments as stock of local biodiversity and showed that integrated systems, such as crop-livestock, are less impact ful to the mesofauna and contribute to the conservation of ant and maintenance of soil quality.

Key words: biodiversity, agroecosystems, soil management.

\section{INTRODUÇÃO}

O cenário atual da produção agropecuária, com o uso intensivo do solo para produzir grãos e carne bovina, pode provocar alterações nos ecossistemas, em várias escalas, principalmente afetando a estrutura trófica da comunidade biológica, além de alterações nos atributos químicos e físicos (MARCHÃO, 2009). As formigas são componentes importantes desta comunidade do solo, principalmente por seus atributos ecológicos, como grande biomassa e atividades que realizam na engenharia de seus ninhos, atuando na aeração do solo e no banco de sementes. As formigas apresentam grande diversidade e atualmente são consideradas14.954 espécies e subespécies válidas, distribuídas em 22 subfamílias em

IUniversidade Federal da Grande Dourados (UFGD), Dourados, MS, Brasil.

IIUniversidade Estadual de Mato Grosso do Sul (UEMS), Cidade Universitária de Dourados, CP 351, 79804-970, Dourados, MS, Brasil.

E-mail: irzo_i@terra.com.br. Autor para correspondência.

IIIEmbrapa Agropecuária Oeste, Dourados, MS, Brasil. Recebido 14.10.11 Aprovado 11.12.13 Devolvido pelo autor 18.03.14 
todo mundo. Para a região Neotropical, são estimadas mais de 4.164 espécies e subespécies distribuídas em 15 subfamílias e 136 gêneros, sendo 14 gêneros e 1.906 espécies endêmicas. Para o Brasil são reconhecidas atualmente 1.456 espécies e 103 gêneros (ANTWIK. ORG; ANTWEB.ORG).

A mirmecofauna tem sido utilizada como bioindicadora da qualidade de sistemas naturais, por apresentar grande complexidade estrutural e sensibilidade às mudanças do ambiente (ANDERSEN, 1997). Com um protocolo de coleta bem estruturado, o uso das formigas como indicadores ambientais é possível graças a ferramentas e processos de espécies acessíveis, sendo muito empregadas em inventários rápidos de diversidade e em monitoramento ambiental (ALONSO, 2000). A descrição da diversidade de Formicidae e dos processos responsáveis pela sua manutenção constitui-se numa importante ferramenta para modelar planos de conservação de ecossistemas naturais ou ameaçados, além de contribuir para estudos biogeográficos, ecológicos e taxonômicos (ANDRÉ et al., 2002; HÖLLDOBLER \& WILSON, 1990).

Na Região Centro-Oeste do Brasil, os sistemas de produção de soja, milho e pastagem cultivada continuamente têm importância destacada na economia nacional e ocupam grande parte da área cultivada. Entretanto, a ocupação da paisagem por agrossistemas não a transforma em um ambiente totalmente inóspito a todas as espécies de formigas. Nesse contexto, o manejo de sistemas agropecuários, com o uso de coberturas vegetais, tem demonstrado benefícios na sustentabilidade e manutenção das propriedades do solo (AQUINO et al., 2008). Contudo, são escassos estudos avaliando parâmetros ecológicos da comunidade de formigas em agroecossistemas, como a integração lavoura-pecuária.

Assim, o presente estudo teve como objetivo avaliar a comunidade de formigas do solo em sistema integrado lavoura-pecuária, comparando diferentes parâmetros ecológicos com a de outros sistemas de manejo, envolvendo agricultura e pecuária, separadamente, além de sistemas naturais da região que incluem a Floresta Semidecidual e o Cerrado.

\section{MATERIAL E MÉTODOS}

O estudo foi realizado na safra 2009/2010, num experimento de longa duração, estabelecido desde 1995, no campo experimental da Embrapa Agropecuária Oeste, em Dourados, MS (22 $14^{\prime} \mathrm{S}$ e $\left.54^{\circ} 49^{\prime} \mathrm{W}\right)$. O clima da região é classificado como Cwa, mesotérmico úmido, com verão quente e inverno seco
(FIETZ \& FISCH, 2008). Os sistemas de manejo do solo foram implantados em um Latossolo Vermelho distroférrico típico, com 70\% de argila (AMARAL et al., 2000).

O sistema integrado lavoura-pecuária (SI) foi estabelecido em faixas, subdividida em duas partes de 4ha, cada, sendo uma ocupada com lavoura e outra com pastagem (Brachiaria decumbens Stapf). Os sistemas com lavoura e pastagem são rotacionados a cada dois anos. Na área ocupada com lavoura, a soja foi conduzida no sistema plantio direto (SPD), no verão, rotacionada com aveia e, no inverno, utilizada como pastagem temporária.

Os sistemas agrícolas avaliados, para comparação, incluíram os manejos sob sistema convencional (aração e gradagem) e sistema plantio direto. O manejo do solo com pastagem cultivada continuamente com B. decumbens foi utilizado como referencial comparativo para aferição da qualidade do solo. Além desses sistemas, foram realizadas amostragens de formigas em fragmentos de Cerrado (CE) (stricto sensu) e de Floresta Semidecídua (FS), adjacente às parcelas experimentais, como referencial para comparação.

O sistema convencional (SC), estabelecido em área de 2ha, consistiu dos cultivos de soja (Glycine max Merril), no verão, e de aveia (Avena strigosa Schreb), no outono/inverno, sendo preparados com uso de grade de disco. O sistema plantio direto (SPD) foi estabelecido numa faixa subdividida em três partes (a, b e c), de 2,8ha cada, sendo duas ocupadas com soja e uma com milho, no verão, rotacionadas com aveia, trigo e nabo forrageiro, durante o outono/inverno. As culturas de trigo e aveia foram utilizadas para produção de grãos, enquanto o nabo forrageiro foi utilizado para produção de palha. O sistema sob pastagem cultivada continuamente (PC) foi estabelecido numa área de aproximadamente 10 ha.

Em cada sistema avaliado, foram definidos cinco pontos de amostragem equidistantes em $30 \mathrm{~m}$, ao longo de um transecto. Em cada ponto, consistindo de uma amostra de $1,0 \mathrm{~m}^{2}$, a serapilheira foi recolhida e peneirada em malha $0,5 \mathrm{~mm}$. O volume recolhido foi transferido para recipientes de malha e dependurados dentro dos extratores do tipo mini-Winkler. Foram realizadas amostragens do solo para caracterização química, de acordo com CLAESSEN (1997), conforme apresentado na tabela 1 . O protocolo de coleta seguiu a padronização feita por AGOSTI \& ALONSO (2000).

As formigas foram montadas com alfinete entomológico, colocadas em triângulos de plástico e etiquetadas em papel vegetal, sendo identificadas 
Tabela 1 - Características químicas do solo, profundidade $0-10 \mathrm{~cm}$, em sistema convencional (SC), sistema integrado lavoura/pecuária (SI-a e SI-b), sistema plantio direto (SPD-a, SPD-b e SPD-c), pastagem contínua (PC), Cerrado (CE) e Floresta Semidecídua (FS).

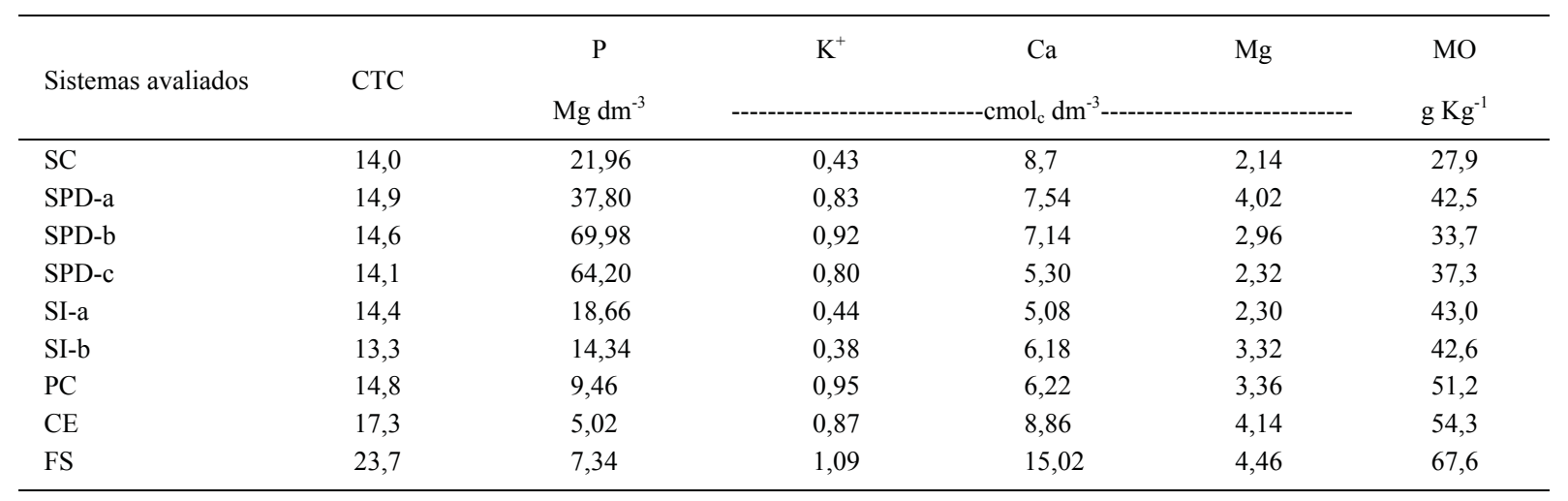

em nível específico, seguindo os guias disponíveis (BOLTON, 1994; FERNANDEZ, 2003; WARD, 2012).

A similaridade entre os diferentes agroecossistemas e os sistemas naturais foi comparada pelo índice de Jaccard: $c j=c /(a+b)-c$, em que $c j=$ índice de similaridade, $\mathrm{a}=$ espécies encontradas no local $\mathrm{a}, \mathrm{b}=$ espécies encontradas no local $\mathrm{b}$ e $\mathrm{c}=$ espécies encontradas em ambos os locais (a e b).

Para análise de diversidade, foi usado o índice de Shannon Wiener: $H^{\prime}=-\sum_{i=1}^{S} p_{i} \ln p_{i}$, em que $\mathrm{H}^{\prime}=$ índice de diversidade, $\mathrm{p}=$ morfoespécies encontradas, $\mathrm{S}=$ riqueza específica.

Para comparar os valores dos índices de diversidade e a quantidade de matéria orgânica presente nos sistemas avaliados, foi realizada uma análise paramétrica de correlação linear de Pearson $(\mathrm{P}<0,05)$. As análises foram realizadas através do programa BioEstat (versão 5.0) (AYRES et al., 2007).

\section{RESULTADOS E DISCUSSÃO}

Foram coletadas 37 morfoespécies de formigas, distribuídas em 23 gêneros e seis subfamílias nos diferentes sistemas avaliados, representando 55 amostras e 625 registros de espécies (Tabela 2). Treze espécies $(56,5 \%)$ foram representadas por apenas um registro em todos os sistemas avaliados, evidenciando a contribuição que as espécies raras apresentam para a composição da biodiversidade. SILVA \& SILVESTRE, (2000), relatam que 28\% da comunidade de formigas amostradas com Winkler é constituída por espécies que são registradas apenas uma única vez (singletons). O significativo número de espécies acidentais, comparado ao total de espécies encontradas, vai ao encontro dos resultados de LUTINSKI \& GARCIA (2005), que argumentam que esta instabilidade pode estar relacionada com fatores ambientais e com a interferência antrópica.
Pode-se observar uma tendência positiva de correlação entre a diversidade da comunidade de formigas e o conteúdo de matéria orgânica $\left(\mathrm{mg} \mathrm{cm}^{-3}\right)$ presente nos sistemas avaliados $(\mathrm{r}=0,7391$ e $\mathrm{P}=0,0228)$ (Figura 1). À medida que aumenta o conteúdo de matéria orgânica no solo, crescem os valores de diversidade (Figura 1). Entre os sistemas com interferência antrópica, verificou-se que o sistema integrado lavoura-pecuária apresentou a maior diversidade de formigas (Figura 2), provavelmente em função da maior disponibilidade de resíduos vegetais provenientes da rotação de lavouras e pastagem. Essa condição pode propiciar condições mais favoráveis quanto à fonte de alimento e habitat para grande parte das espécies de invertebrados que habitam estes solos, aumentando a disponibilidade de energia no sistema (AQUINO et al., 2008).

Através da análise de agrupamento, envolvendo os diferentes agroecossistemas, foi possível observar a formação de dois grupos (A e B), baseados nos índices de similaridade de Jaccard, sendo representados pelos sistemas naturais (grupo A) e sistemas manejados (grupo B), com distância de ligação de $76 \%$ de dissimilaridade, o que permite inferir que a similaridade entre eles é de $24 \%$ (Figura 3). O grupo " $A$ " engloba os ecossistemas naturais (Cerrado e Floresta Semidecídua), com $47 \%$ de similaridade entre si. Nesse grupo, está presente uma fauna invertebrada típica e intimamente relacionada com taxa elevada de táxons especializados de serapilheira, que são sensíveis à cobertura vegetal. $\mathrm{O}$ fornecimento constante e heterogêneo de material orgânico pela vegetação nativa proporciona níveis mais adequados de temperatura e umidade e favorece as condições edáficas necessárias para abrigar uma maior diversidade (AQUINO et al., 2008; SILVA et al., 2006).

No grupo " $\mathrm{B}$ ", observou-se a formação de dois níveis de agrupamentos distintos (B1 e B2) (Figura 3), 
Tabela 2 - Distribuição e riqueza da comunidade de formigas: sistema convencional (SC), pastagem contínua (PC), sistema plantio direto (SPD-a, SPD-b, SPD-c), sistema integrado (SI-a e SI-b), Cerrado (CE) e Floresta Semidecídua (FS).

\begin{tabular}{|c|c|c|c|c|c|c|c|c|c|}
\hline Morfoespécies & $\mathrm{SC}$ & SPD-a & SPD-b & SPD-c & SI-a & SI-b & $\mathrm{PC}$ & $\mathrm{CE}$ & FS \\
\hline Acromyrmex sp. & 0 & 0 & 0 & 0 & 0 & 0 & 1 & 1 & 0 \\
\hline Anochetus diegensis & 0 & 0 & 1 & 1 & 1 & 1 & 0 & 1 & 1 \\
\hline Ambyopone armigera & 0 & 0 & 0 & 0 & 0 & 0 & 0 & 1 & 1 \\
\hline Apterostigma pilosum & 0 & 0 & 0 & 0 & 0 & 0 & 0 & 1 & 0 \\
\hline Camponotus sp. & 1 & 0 & 0 & 0 & 0 & 1 & 0 & 0 & 1 \\
\hline Cephalotes sp. & 0 & 0 & 0 & 0 & 0 & 0 & 0 & 0 & 1 \\
\hline Cyphomyrmex rimosus & 0 & 0 & 0 & 0 & 0 & 0 & 0 & 1 & 1 \\
\hline Crematogaster sp. & 0 & 0 & 0 & 1 & 0 & 0 & 0 & 0 & 0 \\
\hline Hypoponera sp. 1 & 0 & 1 & 1 & 1 & 0 & 1 & 0 & 0 & 0 \\
\hline Hypoponera sp. 2 & 0 & 0 & 0 & 0 & 0 & 0 & 0 & 1 & 1 \\
\hline Hypoponera sp. 3 & 0 & 0 & 0 & 0 & 0 & 1 & 0 & 1 & 1 \\
\hline Hypoponera sp. 4 & 0 & 0 & 0 & 0 & 0 & 0 & 0 & 1 & 0 \\
\hline Hypoponera sp. 5 & 1 & 0 & 0 & 0 & 0 & 0 & 0 & 0 & 0 \\
\hline Ochetomyrmex sp. & 0 & 0 & 0 & 0 & 0 & 0 & 0 & 1 & 0 \\
\hline Octostruma balzani & 0 & 0 & 0 & 0 & 0 & 0 & 0 & 1 & 1 \\
\hline Odontomachus bauri & 0 & 0 & 1 & 0 & 0 & 0 & 0 & 0 & 0 \\
\hline Odontomachus sp. 1 & 0 & 0 & 0 & 0 & 0 & 0 & 0 & 1 & 1 \\
\hline Oligomyrmex sp. & 0 & 0 & 0 & 0 & 1 & 0 & 0 & 0 & 0 \\
\hline Pachycondila ferruginea & 0 & 0 & 0 & 0 & 0 & 0 & 0 & 1 & 0 \\
\hline Pachycondila harpax & 0 & 0 & 0 & 0 & 0 & 0 & 0 & 1 & 1 \\
\hline Paratrechina sp. & 0 & 0 & 0 & 0 & 0 & 0 & 0 & 0 & 1 \\
\hline Pheidole sp. 1 & 1 & 1 & 1 & 1 & 0 & 0 & 1 & 0 & 0 \\
\hline Pheidole sp. 2 & 1 & 1 & 1 & 0 & 1 & 0 & 1 & 0 & 0 \\
\hline Pheidole sp. 3 & 0 & 0 & 0 & 0 & 0 & 0 & 0 & 1 & 1 \\
\hline Pheilde sp. 4 & 0 & 0 & 0 & 0 & 0 & 0 & 0 & 1 & 0 \\
\hline Pseudomyrmex sp. 1 & 1 & 0 & 0 & 0 & 0 & 0 & 1 & 0 & 0 \\
\hline Pseudomyrmex sp. 2 & 0 & 0 & 0 & 0 & 1 & 1 & 0 & 0 & 0 \\
\hline Solenopsis sp. 1 & 0 & 1 & 0 & 1 & 1 & 0 & 0 & 1 & 0 \\
\hline Solenopsis sp. 2 & 0 & 0 & 0 & 0 & 1 & 1 & 1 & 0 & 1 \\
\hline Solenopsis sp. 3 & 0 & 0 & 0 & 0 & 0 & 0 & 0 & 0 & 1 \\
\hline Serycomyrmex sp. 1 & 0 & 0 & 0 & 0 & 0 & 0 & 0 & 1 & 1 \\
\hline Strumygenys sp. 1 & 0 & 1 & 0 & 0 & 1 & 1 & 0 & 1 & 1 \\
\hline Strumygenys sp. 2 & 0 & 0 & 0 & 1 & 0 & 0 & 0 & 1 & 1 \\
\hline Strumygenys sp. 3 & 0 & 0 & 0 & 0 & 1 & 0 & 0 & 0 & 0 \\
\hline Tapinoma sp. 1 & 0 & 0 & 1 & 1 & 0 & 1 & 0 & 0 & 0 \\
\hline Trachymyrmex sp. & 0 & 0 & 0 & 0 & 0 & 0 & 0 & 0 & 1 \\
\hline Wasmmania sp. 1 & 0 & 0 & 0 & 0 & 0 & 0 & 0 & 1 & 0 \\
\hline Riqueza & 5 & 5 & 6 & 7 & 8 & 8 & 5 & 20 & 18 \\
\hline
\end{tabular}

com distância de ligação de $75 \%$ de dissimilaridade. O primeiro nível "B1" apresentou uma semelhança de $31 \%$ entre o sistema integrado lavoura/pecuária (SIa e SI-b) e o sistema plantio direto (SPD-a, SPD-b e SPD-c). Pode-se observar similaridade de $34 \%$ entre os sistemas SI-a e SI-b, com distância de ligação de 69\% do sistema plantio direto (SPD-a, SPD-b e SPD-c). Entre as faixas com os sistemas em plantio direto, verificouse uma similaridade de $37 \%$, sendo que o SPD-b e o SPD-c apresentou 45\% de similaridade (Figura 3). Os sistemas integrados favoreceram o estabelecimento da comunidade de formigas, especialmente alguns grupos hipogeicos de Hypoponera sp. e Strumigenys sp. As formigas são distribuídas em guildas (SILVESTRE et al., 2003) e os gêneros Hypoponera (Poneríneos crípticos predadores generalista) e Strumygenis (Mirmicíneos crípticos predadores especialistas) ocupam funções distintas nas relações tróficas da mesofauna de solo e a presença dessas espécies a partir de uma dinâmica ecológica estabelecida na comunidade por vários níveis tróficos. O conteúdo elevado de matéria orgânica nestes sistemas poderia atuar como atrativo de invertebrados decompositores (Thysanura, Collembola, Oligochaetha e Chilopoda), 


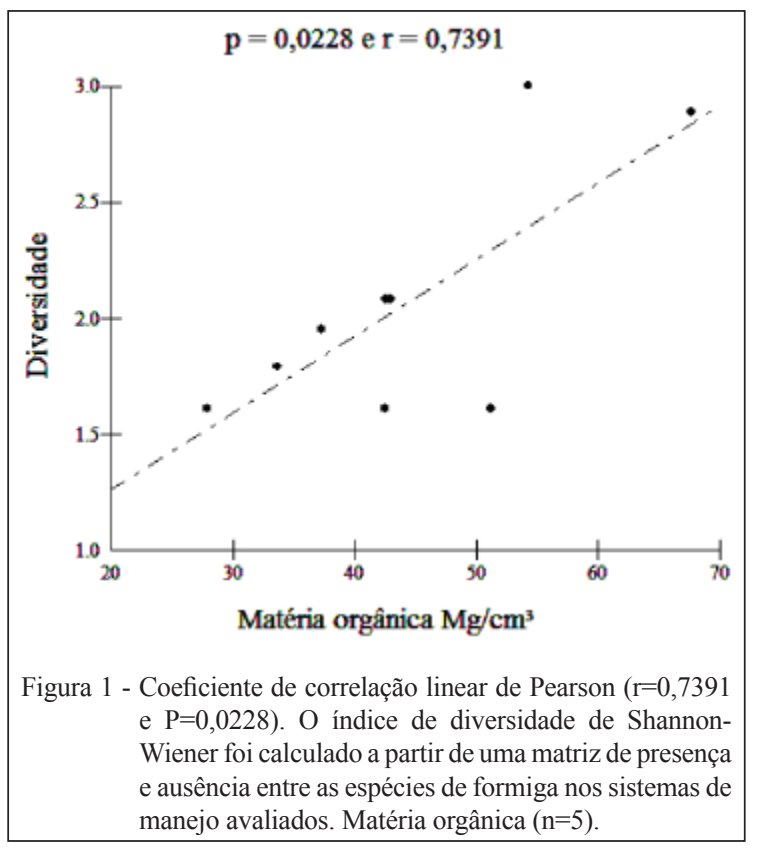

num primeiro estágio, em seguida, surgiriam os predadores (Formicidae, Araneae e ColeOptera), que podem contribuir para o controle natural de insetos nocivos aos cultivos (SILVA et al., 2006).

A similaridade entre o sistema plantio direto e o sistema integrado lavoura-pecuária pode estar relacionada com o sistema de rotação de culturas, que permite o estabelecimento definitivo de colônias de formigas, em ambos os sistemas, de maneira similar e permite que a estrutura da comunidade tenha uma certa estabilidade trófica. Esses sistemas se enquadrariam dentro de um programa de biodiversidade planejada (ALTIERI et

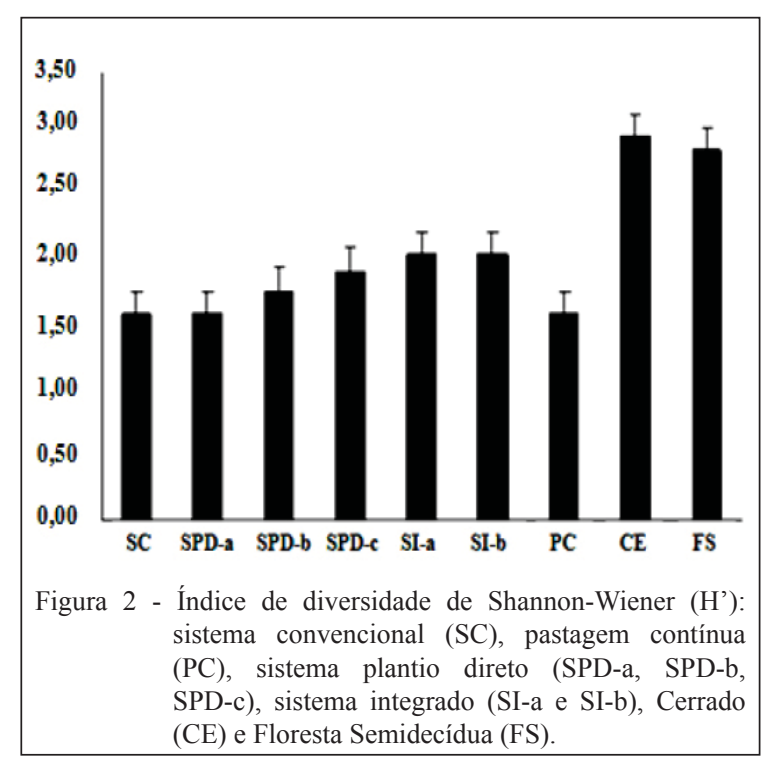

al., 2007), em que o sistema de cobertura enriquece o solo e auxilia o desenvolvimento das culturas de soja e milho. Além disso, com uma função indireta, essa cobertura poderia proporcionar o estabelecimento de colônias de formigas oriundas dos entornos dos ecossistemas do Cerrado e da Floresta Semidecidual.

O segundo nível "B2" englobou o sistema convencional (SC) e o sistema sob pastagem cultivada continuamente (PC), com $43 \%$ de similaridade. Nesses sistemas, as morfo-espécies de Pheidole, Solenopsis e Acromyrmex são comuns, sendo facilmente encontradas em sistemas de produção degradados. Deve-se salientar que o agrupamento do SC com a PC (Figura 3) apresentou a menor riqueza, com cinco espécies cada (Tabela 2). Provavelmente, a baixa diversidade encontrada no $\mathrm{SC}$ pode estar relacionada às modificações impostas pela aração e gradagens, como a destruição do habitat, escassez de alimentos disponíveis e condições de temperatura e umidade desfavoráveis (BARETTA et al., 2006). DIAS et al. (2007) observaram que a rotação de pastagem e leguminosas favoreceu o aumento da diversidade da macrofauna do solo, provavelmente neste estudo a maior diversidade da mirmecofauna ocorreu em função da maior disponibilidade de serapilheira depositada no solo.

As amostras realizadas nas áreas naturais foram bem superiores em termos de riqueza e diversidade de formigas, indicando maior estabilidade estrutural. Esse fato nos leva a pensar que este tipo de estoque adjacente aos sistemas rotacionais é importante para a conservação e reposição dos elementos bióticos.

\section{CONCLUSÃO}

O sistema integrado lavoura-pecuária, rotacionado a cada dois anos, pode favorecer a recomposição da diversidade da comunidade de formigas, consequentemente, estabelecer o equilíbrio dinâmico desses organismos e a manutenção da qualidade do solo. As morfoespécies de Strumygenys sp. e Hypoponera sp. foram encontradas apenas nos manejos de integração lavoura-pecuária e sistema plantio direto. Esses sistemas de manejo podem propiciar condições mais favoráveis para a ocorrência de determinadas espécies de formigas que necessitam de maior estabilidade nas relações tróficas para a coexistência no local.

\section{AGRADECIMENTOS}

Ao PROBIO II (Projeto Nacional de Ações Integradas Público-Privadas para a Biodiversidade), pelas bolsas e suporte financeiro concedidos; aos laboratoristas da Embrapa Agropecuária 


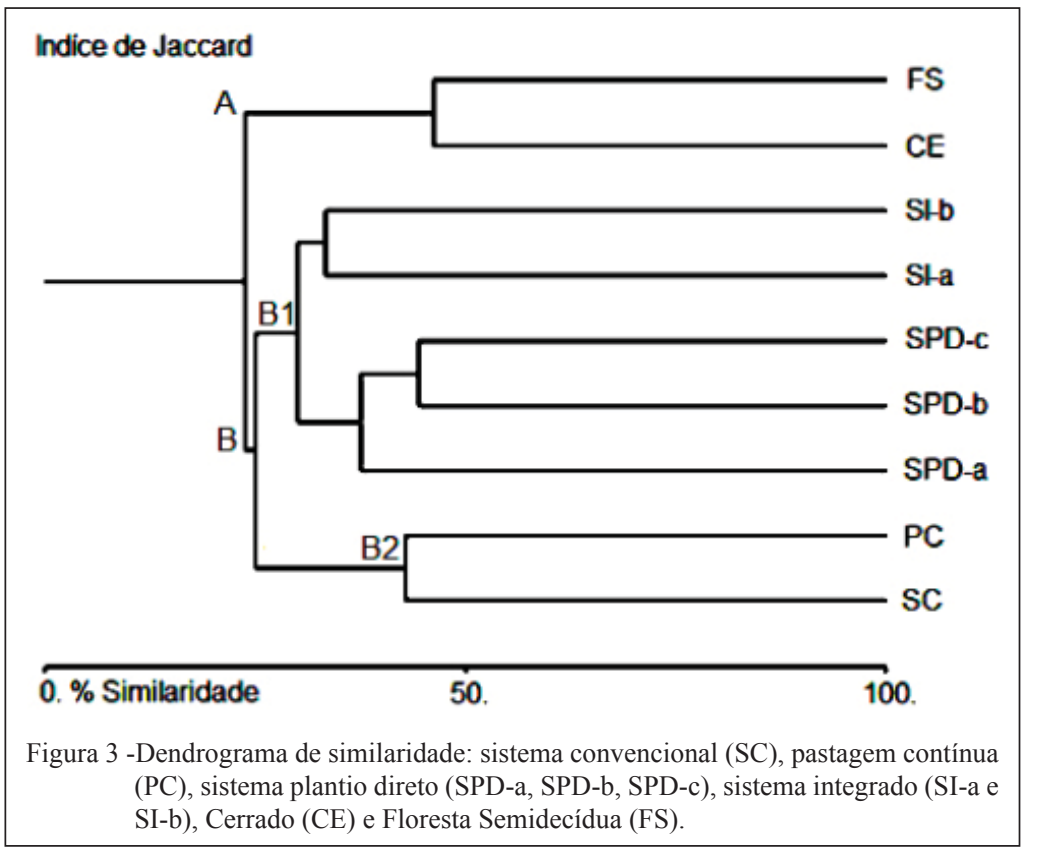

Oeste, pelo auxílio nas análises físicas e químicas. Ao Laboratório de Ecologia de Hymenoptera (Hecolab) da UFGD. Fábio Martins Mercante agradece ao $\mathrm{CNPq}$, pela bolsa de Produtividade em Pesquisa.

\section{REFERÊNCIAS}

ANTWIKI.ORG. Antwiki. Acesso em: 05 set. 2011. Online. Disponível em: <http://antwiki.org/>.

ANTWEB.ORG. Antweb. Acesso em: 08 set. 2011. Online. Disponível em: $<$ http://antweb.org/>.

ALONSO, L.E. Ants as indicator of diversity. In: AGOSTI, D. et al. (Eds.). Standard methods for measuring and monitoring biodiversity. Washington and London: Smithsonian Institution, 2000. 280p.

ALTIERI, M.A. et al. Manejando insetos-praga com a diversificação de plantas. Agriculturas, v.4, n.1, p.20-23, 2007. Disponivel em: <http://subscriptions.leisa.info/index.php?url=article-details. tpl\&p\%5B_id\%5D=91256>. Acesso em: 01ago. 2011.

AMARAL, J.A.M. et al. Levantamento semidetalhado dos solos do campo experimental de Dourados, da Embrapa Agropecuária Oeste, Município de Dourados, MS. Dourados: Embrapa Agropecuária Oeste; Rio de Janeiro: Embrapa Solos, 2000. 68p. (Embrapa Agropecuária Oeste. Documentos, 22; Embrapa Solos. Documentos, 15).

ANDERSEN A.N. Using ants as bioindicators: multiscales issues in ant community ecology. Conservation Ecology, v.1, n.1, p.821, 1997. Disponível em: <http://www.consecol.org/vol1/iss1/ art8/>. Acesso em: 01out. 2011.

ANDRÉ, H.M. et al. Soil biodiversity: mith, reality or conning? Oikos, v.96, p.3-24, 2002. Disponível em: <http://onlinelibrary. wiley.com>. Acesso em: 05 set. 2011. doi: 10.1034/j.16000706.2002.11216.x.
AQUINO, A.M. et al. Invertebrate soil macrofauna under different ground cover plantes in the no-till system in the Cerrado. European Journal of Soil Biology, v.44, p.91-97, 2008. Disponível em: <http://www.sciencedirect.com/science/article/pii/ S1164556307000465>. Acesso em: 20 ago. 2011. doi:10.1016/j. ejsobi.2007.05.001.

AYRES, M. et al. BioEstat. Versão 5.0, Sociedade Civil Mamirauá, MCT - CNPq, Belém, Pará, Brasil, 2007. Disponível em: $<$ http://bioestat.software.informer.com/5.0/>. Acesso em: 21 jun. 2011 .

BARETTA, D. et al. Efeito do cultivo do solo sobre a diversidade da fauna edáfica no planalto sul catarinense. Revista de Ciências Agroveterinárias, v.5, n.2, p.108-117, 2006. Disponível em: <http:// rca.cav.udesc.br/index.php?ID=5>. Acesso em: 10 ago. 2011.

BOLTON B. Identification guide to the ant genera of the world. London: Harvard University, 1994. 222p.

CLAESSEN, M.E.C. (Org.). Manual de métodos de análise de solo. 2.ed. Rio de Janeiro: Embrapa-CNPS, 1997. 212p.

DIAS, P.F. et al. Efeito de leguminosas arbóreas sobre a macrofauna do solo em pastagem de Brachiaria brizantha $\mathrm{cv}$. Marandu. Pesquisa Agropecuária Tropical, v.37, p.38-44, 2007. Disponível em: <http://www.revistas.ufg.br/index.php/pat/article/ view/1863>. Acesso em: 21 ago. 2011.

FERNANDEZ, F. Introducción a lashormigas de laregión Neotropical. Bogotá, Colômbia: Instituto de Investigación de Recursos Biológicos Alexander von Humboldt, 2003. 398p.

FIETZ, C.R.; FISCH, G.F. O clima da região de Dourados, MS. 2.ed. Dourados: Embrapa Agropecuária Oeste, 2008. 32p. (Embrapa Agropecuária Oeste. Documentos, 92).

HÖLLDOBLER, B.; WILSON, E.O. The ants. Cambridge, Massachusetts: Belknap, 1990. 732p. 
LUTINSK, J.A.; GARCIA, F.R.M. Análise faunística de Formicidae (Hymenoptera: Apocrita) emecossistema degradado no municípiode Chapecó, Santa Catarina. Biotemas, v.18, n.2, p.73-86, 2005.

MARCHÃO, R.L. et al. Soil macrofauna under integrated crolivestock systems in a Brazilian Cerrado Ferralsol. Pesquisa Agropecuária Brasileira, v.44, n.8, p.1011-1020, 2009. Disponível em: <http://webnotes.sct.embrapa.br/pab/pab.nsf/ FrAnual>. Acesso em: 21 ago. 2011.

SILVA, R.F. et al. Macrofauna invertebrada do solo sob diferentes sistemas de produção em Latossolo da região do cerrado. Pesquisa Agropecuária Brasileira, v.41, p.697-704, 2006. Disponível em: $<$ www.scielo.br/pdf/pab/v41n4/29819.pdf>. Acesso em: 02 set. 2011.
SILVA, R.R.; SILVESTRE, R. Diversidade de formigas (Hym: Formicidae) em Seara Santa Catarina. Biotemas, v.13, n.2, p.85$105,2000$.

SILVESTRE R. et al. Grupos funcionales de hormigas: el caso de los gremios del Cerrado. In: FERNANDEZ F. (Ed.). Introducción a las hormigas de la región Neotropical. Bogotá: Instituto de Investigación de Recursos Biológicos Alexander von Humboldt, 2003. p.113-148.

WARD, P.S. Antweb. Ants of California, 2012. Disponível em: $<$ http://www.antweb.org/california.jsp $>$. Accesso em: $07 \mathrm{fev}$. 2013. 